\title{
Realistic metamaterial lenses: Limitations imposed by discrete structure
}

\author{
M. Lapine, ${ }^{1}$ L. Jelinek, ${ }^{2}$ M. J. Freire, ${ }^{1}$ and R. Marqués ${ }^{1}$ \\ ${ }^{1}$ Department of Electronics and Electromagnetism, Faculty of Physics, University of Seville, 41012 Seville, Spain \\ ${ }^{2}$ Department of Electromagnetic Field, Czech Technical University in Prague, 16627 Prague, Czech Republic
}

(Received 16 July 2010; published 28 October 2010)

\begin{abstract}
We study the peculiarities of a metamaterial "superlens," caused by its discrete structure and finite size. We show that precise modeling of the lens provides remarkable distinctions from continuous medium approximation. In particular, we address the problem of highest resolution that can be achieved with a realistic electrically thin metamaterial lens. We conclude that discrete structure imposes essential limitations on the resolution and that the resolution cannot be improved by decreasing dissipation in the system. Further implications related to effective medium description of discrete structures are discussed.
\end{abstract}

DOI: 10.1103/PhysRevB.82.165124

PACS number(s): 81.05.Xj, 41.20.-q, 78.67.Pt, 87.61.-c

\section{INTRODUCTION}

Metamaterials attract heaps of research attention for the last 10 years. ${ }^{1,2}$ Although there is no consensus on the very definition of metamaterials, ${ }^{3,4}$ the number of publications rises exponentially and ever new directions are proclaimed promising. A major flow of work and speculations is related to the idea of super-resolution with the so-called "perfect lens," 5 which is perhaps among the few key triggers 6 of the research outburst. While it was soon apparent that the perfect lens is not so perfect, ${ }^{7-9}$ the overall idea appeared to be fruitful enough to be developed up to a level of applications. In particular, metamaterials based on split-ring resonators were put forward for various superlenses. ${ }^{10,11}$

Practical solutions are so far available at microwave and radio frequencies, with the key goal to aid at magnetic resonance imaging (MRI). A metamaterial superlens suggested ${ }^{11}$ for MRI applications employs an idea of imaging with a sufficiently subwavelength slab having negative permeability or permittivity. ${ }^{5}$ This must be distinguished from another approach, based on a multiple point-to-point channelling (canalization), as can be achieved with wires" 12 or "Swiss rolls,"13 although such method is also applicable for MRI. ${ }^{13,14}$ With the split rings, on the other hand, other MRI enhancements are possible, being, for example, the eased detection with flexible ring resonators, ${ }^{15}$ or parametric amplification of MRI signals. ${ }^{16}$

While the latter practical devices allow for a precise and exact theoretical description (in terms of accounting for their structure and all the few elements explicitly), larger metamaterials are normally analyzed with the help of effective medium modeling, ${ }^{17,18}$ so that split-ring structures can be described with effective permeability. ${ }^{19-21}$ Since for a thin lens (comprising just a few structural units in one direction) the effective permeability approach is not directly applicable, a specific continuous slab model was developed ${ }^{22}$ which could be used to calculate transmission/reflection properties with the only approximation that the lens is assumed homogeneous and infinite in transverse directions. Although the latter model was quite efficient to predict the overall performance of the lens, ${ }^{22,23}$ it was soon noticed ${ }^{24}$ that some of the lens properties can differ remarkably whether evaluated with a continuous medium approximation, or assessed experimen- tally. In order to achieve better accuracy in theoretical description, we should follow an explicit approach (see, e.g., Ref. 25) which takes into account interaction between all the elements in a finite structure. Preliminary comparison ${ }^{24} \mathrm{re}$ vealed that the predictions of the discrete model are quite close to the experimental findings while deviate sometimes from the continuous model approximation.

The aim of this paper is to analyze in detail what is the consequence of the discrete structure of the lens and its finite size; how this affects the general lens properties, such as imaging frequency, transmission between the MRI coils, etc.; and how the continuous model can be modified in order to describe some of such features.

\section{SETUP OF THE PROBLEM}

Geometry of the problem and the corresponding discrete modeling approach were described in great detail in a previous publication. ${ }^{24}$ Here we only repeat the most general information as long as necessary for discussing the results.

The isotropic metamaterial lens proposed in Ref. 11 is composed of capacitively loaded rings (CLRs) periodically arranged in an isotropic three-dimensional lattice with the lattice constant $a=1.5 \mathrm{~cm}$. The lens has three planes of 18 by 18 CLRs interlayered with orthogonal segments providing two (mutually orthogonal) sets of two layers 17 by 18 CLRs each, which makes it up to roughly 2200 CLRs. Overall dimensions of the lens are thus $18 \times 18 \times 2$ lattice constants, although it must be noted that "unit cells" are incomplete at the edges of the structure and that there are neither two nor three unit cells across the lens.

The CLRs have the mean radius $r_{0}=0.49 \mathrm{~cm}\left(2 r_{0} / a\right.$ $=0.66)$ and are loaded with lumped nonmagnetic capacitors so that the frequency of resonance in free space equals to $63.28 \mathrm{MHz}\left(k_{0} a=0.02\right)$. The total resistance has been estimated as $R=0.0465 \mathrm{Ohm}$ (obtained indirectly by measurements of the quality factor of the resonator).

We define a Cartesian coordinate system $(x, y, z)$ so that the lens geometrical center is placed at the coordinate origin; the $y$ axis is perpendicular to the lens as slab (lens axis) while the long edges of the lens are parallel to $x$ and $z$ axes (Fig. 1). The lens is thus completely symmetric with respect to the coordinate origin, all the axes and all the coordinate 


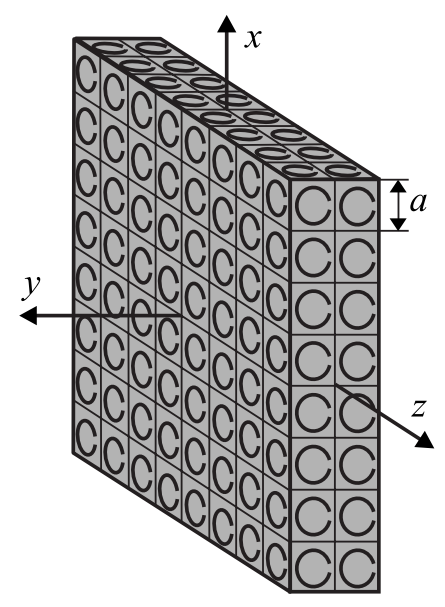

FIG. 1. Scheme of the lens with the corresponding coordinate system. Note that the actual number of elements along $x$ and $z$ directions is not reflected in this sketch.

planes (we neglect minor asymmetry occurring in the real lens caused by specific assembly details such as resulting from substrate thickness; these deviations are of the same order as unavoidable production inaccuracy). The so-called input and output surfaces of the lens correspond to $y$ $=\mp 1.5 \mathrm{~cm}$ while the theoretical source and image planes are $x z$ planes at $y=\mp 3.0 \mathrm{~cm}$.

The discrete analysis of the systems of resonators is well appreciated in literature, particularly in studying magnetoinductive waves in CLR arrays. ${ }^{25-28}$ It constitutes in solving the matrix multi-impedance equation

$$
\overline{\mathbf{Z}} \cdot \mathbf{I}=-j \omega \Phi^{\mathrm{ext}}
$$

with $Z_{n n}=Z_{0}$ and $Z_{n m}=j \omega M_{n m}$. The self-impedance $Z_{0}=R$ $+j \omega L+1 /(j \omega C)$ is determined by the resistance $R$, selfinductance $L$ and self-capacitance $C$ of a single CLR. Here, $\boldsymbol{\Phi}^{\text {ext }}$ represents the total external magnetic flux through the corresponding ring while the mutual interaction between all the rings is expressed in terms of mutual inductances $M_{n m}$. Solving the system (1) for currents with a given distribution of external sources of magnetic field provides the complete description of the lens, as all the interesting characteristics, such as impedance measured by the coil, spatial distribution of the magnetic field produced by the lens, can be calculated as soon as all the currents $I_{n}$ are known. Further specific details of such analysis applied to a large lens are given in. ${ }^{24}$

\section{RESULTS AND DISCUSSION}

\section{A. Imaging frequency}

Within the continuous medium approach, it is well known ${ }^{2}$ that the best imaging with a slab having negative effective permeability occurs at a frequency where $\mu=-1$. For a discrete lens of a finite size, however, the question is less trivial as it is too small to be described with effective parameters. ${ }^{29}$ The assessment can be attempted through some indirect characteristics. Continuous modeling approach suggests ${ }^{2}$ that at the imaging frequency impedance measured with a sufficiently large coil placed at the source plane has a
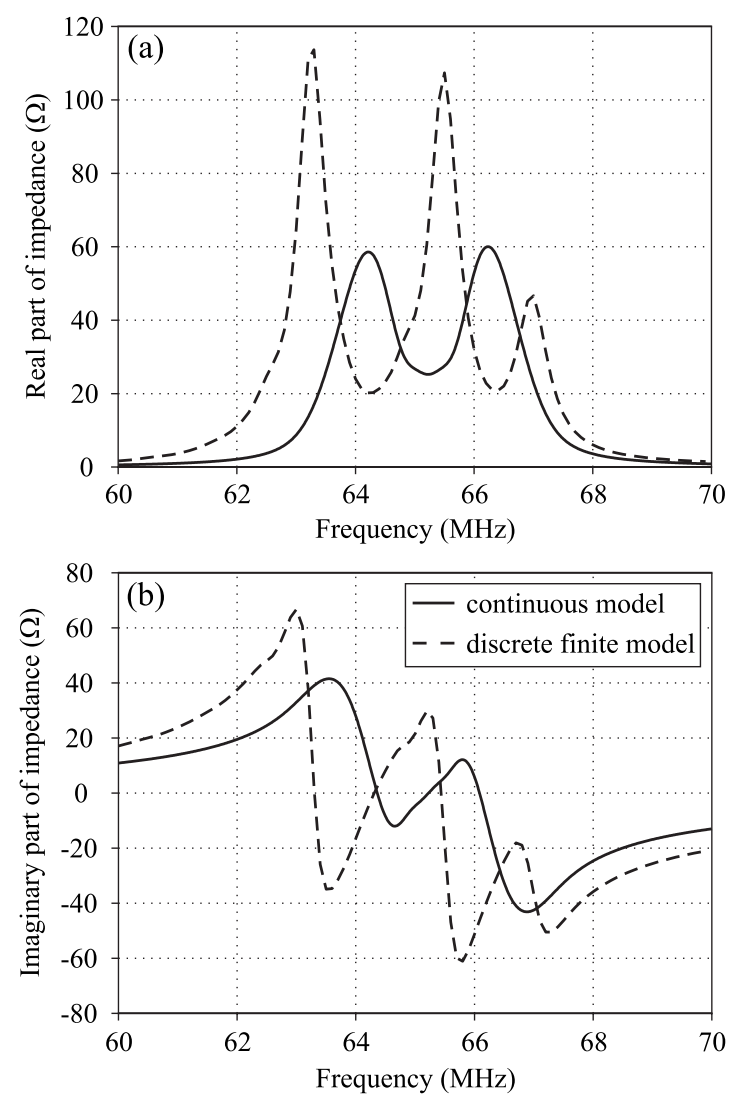

FIG. 2. (a) Real and (b) imaginary parts of impedance for a 5-inch coil placed in the source plane $(15 \mathrm{~mm}$ from the lens surface): comparison between a slab of continuous medium (solid) and discrete model (dash).

local minimum (between the two peaks) in the real part while the imaginary part is crossing zero (Fig. 2, solid curves). However, discrete model predicts a different pattern (Fig. 2, dash curves), expressing a more complex picture of peaks.

Further complication is that with the discrete model, the impedance frequency dependence is not the same for the coils of different size and also depends on the distance between the coil and the lens. With an increase in the coil size, the frequency dependence of the impedance changes qualitatively (Fig. 3). For small coils, a peak at $63.2 \mathrm{MHz}$ (close to the resonance frequency of the corresponding bulk medium) is dominant in the resistance. For moderate coil sizes (5a-10a in diameter), second peak at $65.5 \mathrm{MHz}$ emerges, although the main resonance is still present. For larger coils, this second peak starts to vanish while the third one, at 67 $\mathrm{MHz}$, becomes stronger and for coils larger than $20 a$ is clearly dominant.

These observations imply that the lens shows distinct properties caused not only by the discrete structure but also by its finite size. For this reason, it is only possible to use the corresponding criterion for the coils of moderate size: large enough to make an averaging over many unit cells but still sufficiently small not to be affected by the lens size limitations. From Fig. 3, we can conclude that for moderate coil sizes, (first) local minimum of the resistance occurs within 64.0-64.5 MHz, and the zero crossing of the reactance hap- 

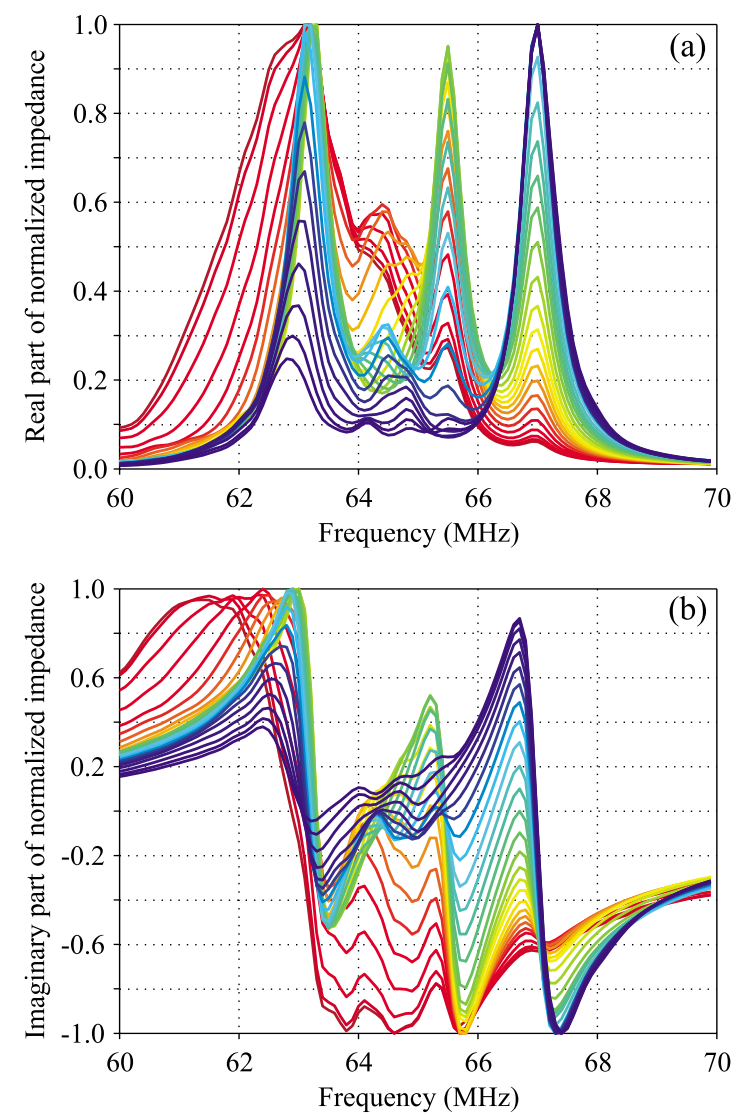

FIG. 3. (Color online) (a) Real and (b) imaginary parts of impedance for coils of different size, placed in the source plane. The color sequence from red to blue (curves upwards at $66.5 \mathrm{MHz}$ ) corresponds to the coils from 1 to $13 \mathrm{~cm}$ in radius, with a step of 0.5 $\mathrm{cm}$. The data are renormalized to have a unity maximum.

pen within the same frequency interval, although we must note that the two characteristic frequencies do not exactly coincide for each particular coil radius.

Similar conclusions can be made by studying the impedance measured by a coil placed at various distances from the lens (Fig. 4). Apart from the case when the coil is too close to the lens surface (when close proximity of some lens rings to the coil apparently has a dominating effect on the pattern of frequency dependence), we can see that characteristic frequencies converge within the interval mentioned above. For this particular coil size (5 inch diameter), the frequency of 64.2-64.4 MHz can be assumed.

Another approach to evaluate the imaging frequency (or, as it is likely, frequency range) lies in the calculation of the transmission between the two identical coils, placed in the source and image planes, respectively. ${ }^{9}$ An easy measure of this quantity can be the ratio of the current induced in the receiving coil, to that in the transmitting coil. For a continuous slab at the imaging frequency (not shown), this ratio has a local minimum (equal to unity in a lossless case); naturally, this occurs exactly at the same frequency as the minimum in the resistance measured by the coil, and zero reactance. ${ }^{22}$ The imaginary part of the current ratio is zero, as there is no phase shift between the two currents. However, the discrete model reveals different observations. Figure 5 shows the ab-
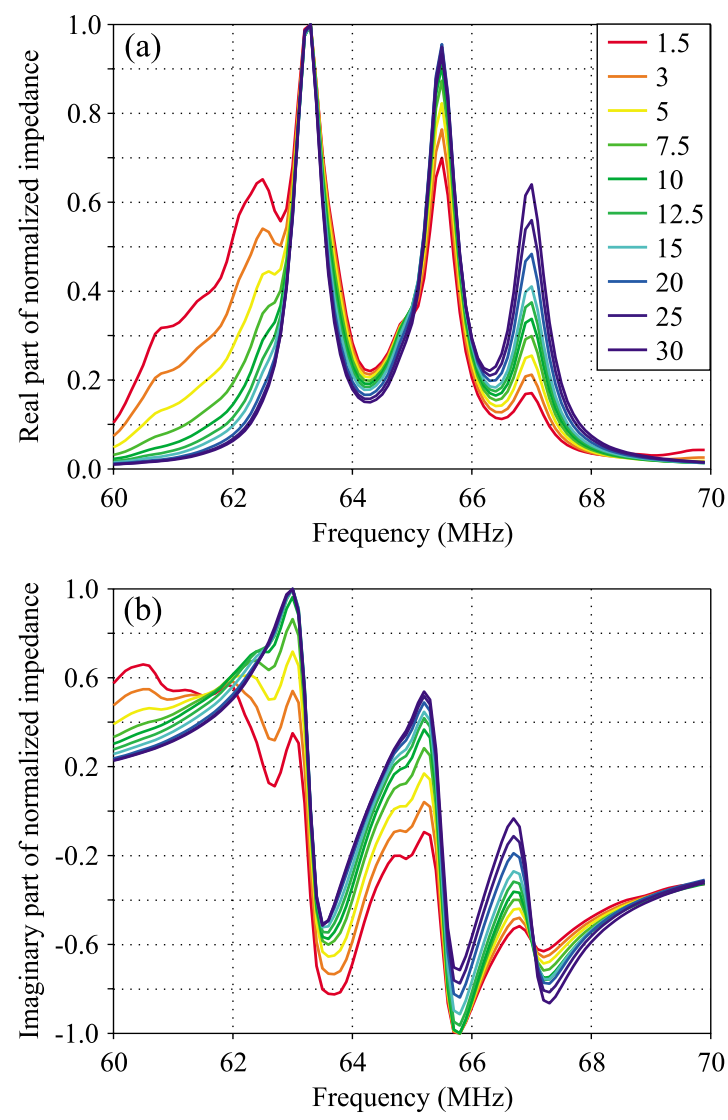

FIG. 4. (Color online) (a) Real and (b) imaginary parts of impedance for a 5-inch coil, placed at various distances from the lens surface (millimeter, shown in the inset). The data are renormalized to have a unity maximum.

solute value and imaginary part of this ratio for different coil sizes. Comparing the absolute values of transmission for different coils, we can admit that while the data for small and large coils vary considerably, there is certain uniformity (aggregation of curves at a local minimum around $64 \mathrm{MHz}$ ) for intermediate coil sizes, $6 a-10 a$ in diameter. This local minimum roughly corresponds to the zero crossing of the imaginary part (renormalized to a unity maximum in Fig. 5 for clarity). For the representative coil sizes mentioned above, the frequency of zero crossing varies between 63.5 and 63.7 $\mathrm{MHz}$, and this frequency range can be assumed to be the result of the transmission criterion for imaging. Note that this result differs from the impedance approach.

So far, we were discussing indirect criteria to evaluate the imaging frequency. The necessity to employ such criteria, in spite of the ambiguity discussed above, is driven by the fact that direct observations of the field patterns produced by the lens from various sources, might be equally unclear. Indeed, as the resolution of the lens is still to be determined (see Sec. III B), we cannot be certain about choosing an appropriate geometry to check if an image can be observed at some frequency. For example, the field patterns observed in the imaging plane for various coils were not found to reproduce the source field in detail at any frequency between 60 and 70 $\mathrm{MHz}$ while a clear central spot could be observed several times across the frequency sweep, interchanged with more 

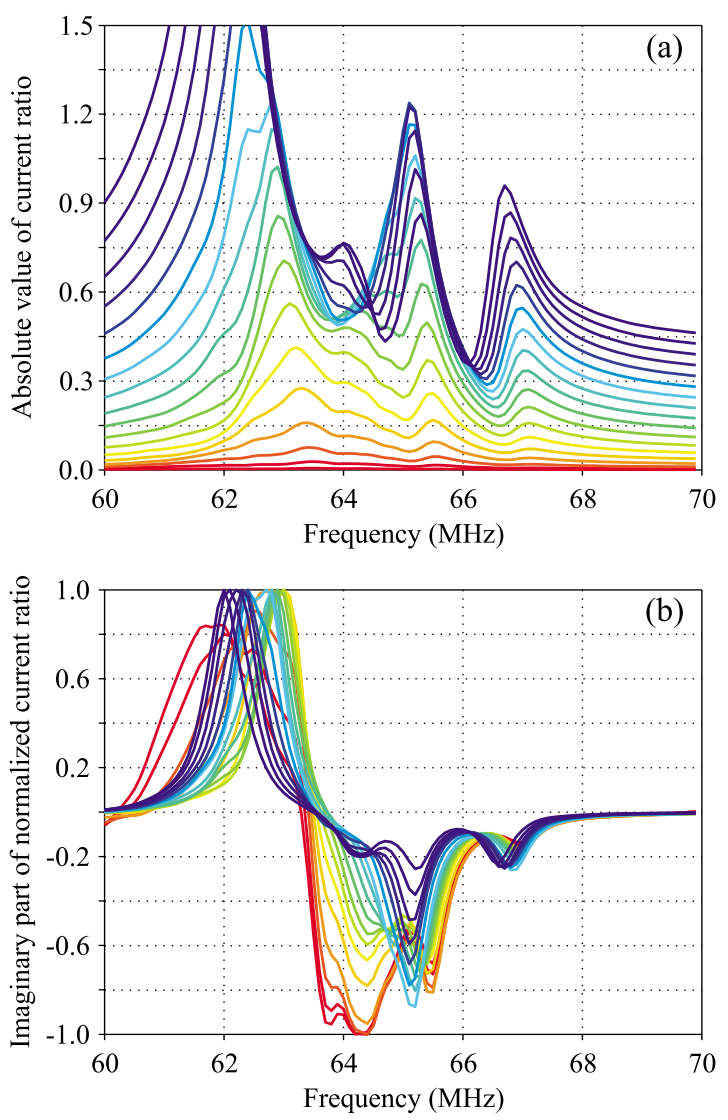

FIG. 5. (Color online) (a) Absolute value and (b) normalized imaginary part of the ratio between the current induced in a coil in the image plane, to the current in the identical coil in the source plane, for coils of different size. The color sequence from red to blue (curves upwards at $64 \mathrm{MHz}$ ) corresponds to the coils from 1 to $9.5 \mathrm{~cm}$ in radius with a step of $0.5 \mathrm{~cm}$.

complex patterns of excitations (data not shown). For the reasons explained in Sec. III B, it appears more reasonable to try an arrangement of two sufficiently distant small sources: two coils of $5 \mathrm{~mm}$ radius positioned symmetrically at $7 a$ distance. Detailed observation of the changes in the field pattern with frequency, illustrated by an animation ${ }^{30}$ made with a $0.1 \mathrm{MHz}$ step in the $60-70 \mathrm{MHz}$ interval, demonstrates that the pattern obtained around $63.6 \mathrm{MHz}$ appears most clear, although we should note that none of the patterns actually correspond to the source field of the small loops. The frequency found this way, is consistent with the criterion based on the transmission between two coils but deviates from the impedance criterion (at $64.0-64.5 \mathrm{MHz}$, further maxima emerge in the field pattern and overall image is less clear). However, we should note that the frequency difference is minor, so for experimental evaluation both the criteria appear to be fairly suitable.

One may argue that a further discrepancy lies in the fact that, contrary to the continuous model which predicts the imaging frequency to be symmetrically between the two resonances of the slab (Fig. 2, solid curves), the frequency we choose is much closer to the first resonance. This is, however, consistent with the findings of paper, ${ }^{22}$ where it was demonstrated that the imaging frequency of the thin slab
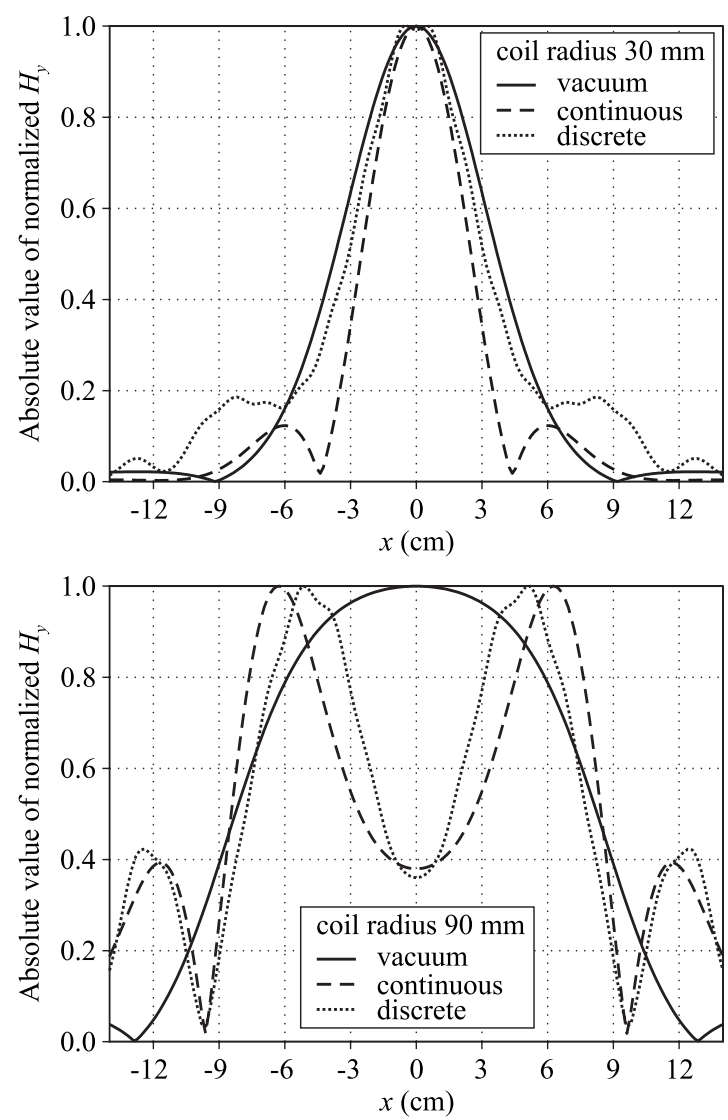

FIG. 6. Spatial profile of the axial component $\left(H_{y}\right)$ of the total magnetic field observed across the image plane $(y=3 \mathrm{~cm}, z=0)$ for a small or a large coil placed in the source plane. Comparison between the field obtained with a discrete lens (at $63.6 \mathrm{MHz}$ ) with a continuous slab (with $\mu=-1$ ) and in vacuum.

is indeed expected to be closer to the first resonance. We therefore should not seek to find imaging phenomena between the second and third peaks of the impedance predicted by discrete model, even though some of the field patterns might look promising.

We should also comment that one further qualitative criterion for a superlens behavior could have been the observation of evanescent field enhancement. However, the field structure inside the lens is strongly nonuniform and cannot show a clear analogy to a field inside a homogeneous slab. At the same time, field analysis in the outer areas faces the same difficulty: there is no unique frequency to be selected with such criterion, as the best visual analogy (field decay from the source and a subsequent decay, from a larger value, on the other side) occurs at different frequencies depending on the coil size and distance to the lens.

All this analysis suggests that, although no direct coincidence between the continuous model and the discrete calculation can be found, the general criteria formulated with the help of continuous model are still applicable. Although we cannot select an exact frequency, ideal for imaging, we can safely speak of a relatively narrow frequency interval-63.5 to $63.7 \mathrm{MHz}$ - where the phenomena, analogous to the imaging with a continuous $\mu=-1$ slab, can be observed with a discrete finite lens. Since the field patterns do not change 
$1 \mathrm{a}$

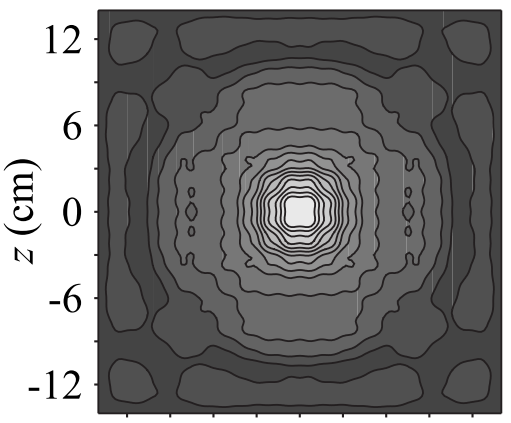

$6 a$

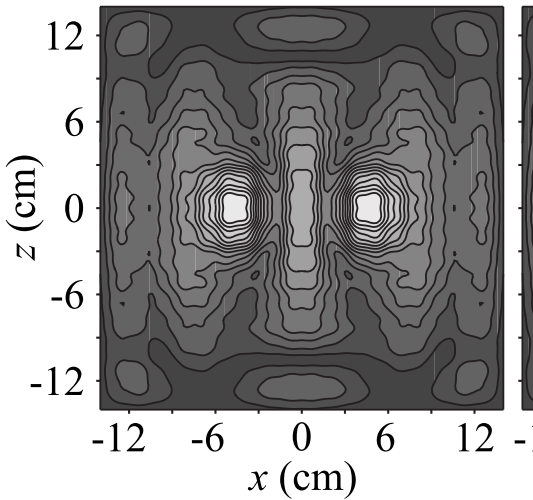

$3 a$

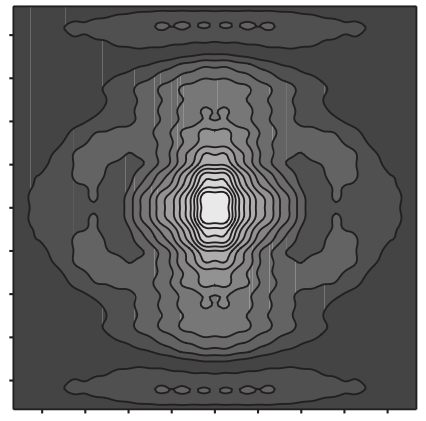

$7 \mathrm{a}$

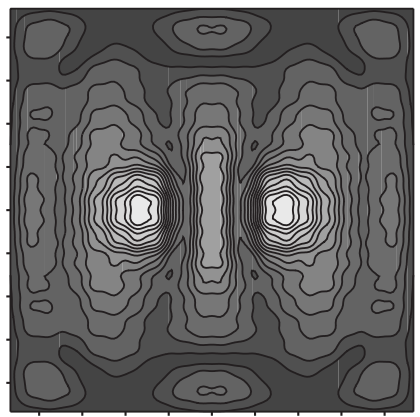

$4 \mathrm{a}$

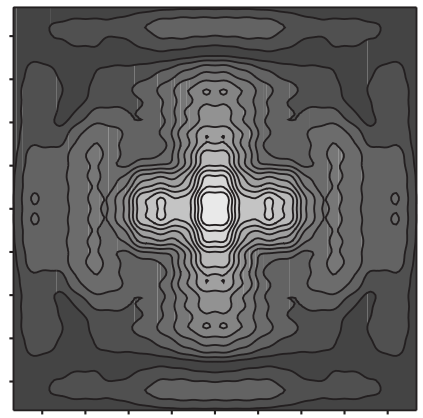

$9 \mathrm{a}$

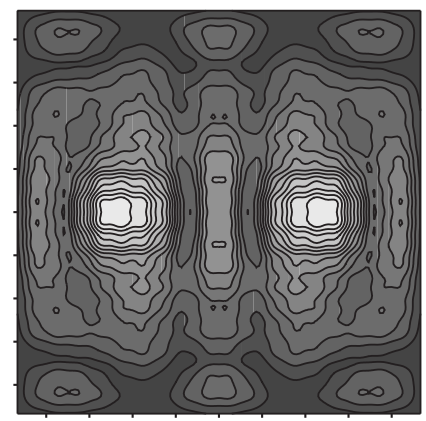

$5 a$

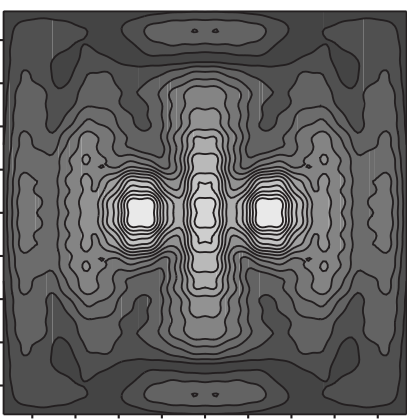

$12 \mathrm{a}$

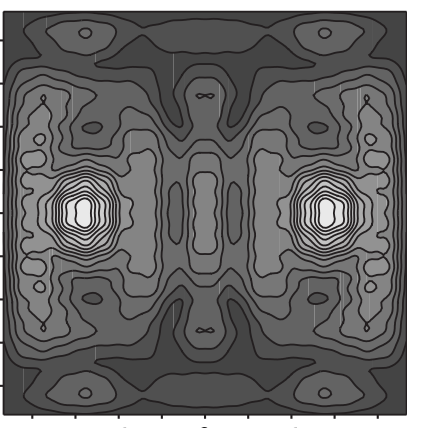

FIG. 7. Axial component $\left(H_{y}\right)$ of the total magnetic field observed at $63.6 \mathrm{MHz}$ in the image plane for the two small sources (coils of 5 $\mathrm{mm}$ radius) placed in the source plane at various distances $(1 a-12 a)$ between each other.

remarkably within this frequency range, we have chosen 63.6 $\mathrm{MHz}$ in order to convey calculations necessary to evaluate the spatial resolution of the lens, analyzed in the following section.

\section{B. Spatial resolution of the lens}

Now that we are confident that the lens behavior corresponding to the imaging phenomena can be reliably studied at frequencies discussed in the previous section, we are able to proceed to the important question of the resolution that can be achieved by the lens. Within the continuous theory, resolution is limited by lens thickness and by dissipation, according to the relation ${ }^{2}$

$$
\Delta=\frac{2 \pi d}{\ln (2 / \delta)} .
$$

For the lens under consideration, having loss tangent $\delta=0.2$ and thickness $d=2 a$, resolution is thus limited by $5.5 a$. This value might be not a good starting point to study the resolution of a discrete structure if we expect it to be of the order of a few lattice constants. Nevertheless, it is interesting to check the behavior of the realistic lens with this respect.

As it was already noticed, ${ }^{24}$ magnetic field distributions behind the lens excited by relatively small coils, are visually rather similar regardless of the coil size. Now, we have systematically studied the patterns in the image plane for coils of different radii, and conclude that the field profiles are practically identical for small coils up to $5 \mathrm{~cm}$ in radius.
These profiles are characterized by $4.3 a$ full-width at halfmaximum, so are almost as wide as the coil field in vacuum (in the absence of the lens) for same coils (Fig. 6). For coil radii larger than $6 \mathrm{~cm}$, the profiles obtained with the lens tend to give a clearly better spot size accuracy than in vacuum. On the other hand, even for larger coils the image field remains unresolved in details, demonstrating only a central spot (of appropriate size) but not the source profile. Only with a coil as large as $9 \mathrm{~cm}$ radius further features in the profile are visible, which are qualitatively analogous to the source field, as also with the continuous model (Fig. 6). Going to much larger sizes is not appropriate as the size of coil becomes comparable with the lens dimensions, and edge effects are be essential, deteriorating the image (not shown). For checking bigger coils, studying a much larger lens would be necessary, which is numerically challenging but will be probably done in the future.

Clearly, the above observations cannot be considered entirely appropriate for understanding the resolution. More consistent approach to test the resolution limitations is to analyze if the lens can distinguish the two point sources with various spacing. As we know that the field patterns obtained with coils smaller than four lattice constants in diameter are practically identical, we can safely choose a coil with the same radius as the ring inside the lens, as a fair representative of a point source. Placing two such coils at various distances between each other in the source plane provides the field patterns in the image plane as shown in Fig. 7. We can see that indeed coils close enough provide either one central spot or a complex pattern which does not reflect actual loca- 


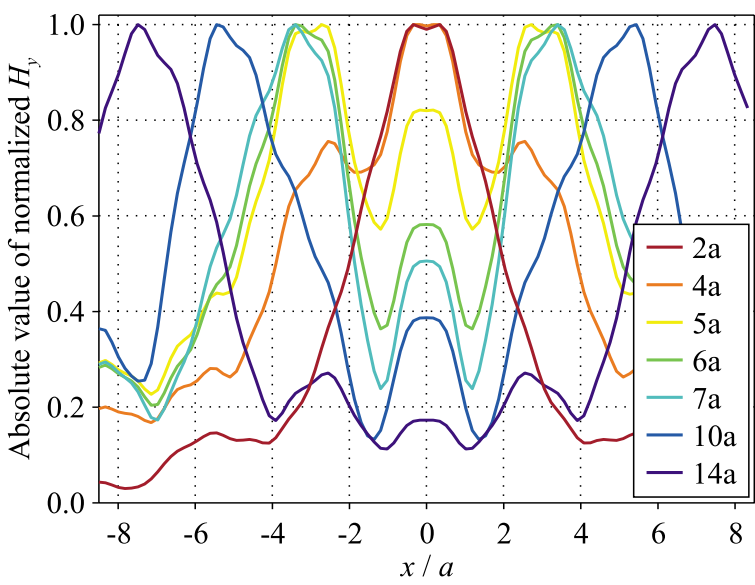

FIG. 8. (Color online) Profiles of the axial component $\left(H_{y}\right)$ of the total magnetic field observed at $63.6 \mathrm{MHz}$ along $x$ axis in the image plane (at $y=3 \mathrm{~cm}, z=0$ ) for the two sources at various distances between each other. The data are renormalized to have a unity maximum.

tion of the sources unambiguously. With separation of $6 a$, the two spots can be distinguished although the "spurious" maximum between them is still strong; for separations larger than $7 a$, resolution is apparent, although of course not the size of the spots but only location corresponds to the source geometry. This can be also conveniently seen on a field profile across the line of the source images in the image plane (Fig. 8). Note, though, that the shape of these profiles makes a standard half-maximum criterion for peak separation barely applicable.

In spite of these complications, we can nevertheless conclude that the spatial resolution of the lens is limited by 5-7 lattice constants. This conclusion can be supported with the continuous model. Indeed, if we force certain truncation for the $k$ vectors which can be transmitted by the lens, we can obtain the field patterns for the corresponding resolution. Figure 9 shows the difference between the virtually unrestricted case $\left(k \leq 300 \mathrm{~m}^{-1}\right.$, corresponding to $1.5 a$ resolution), and the truncated calculation $\left(k \leq 65 \mathrm{~m}^{-1}\right.$, which corresponds to a restriction by $6.5 a$ ). The difference is clearly visible: complete separation of the source spots occurs starting from about $4 a$ in the first case but only at $6 a$ in the second. This test supports indirectly the conclusion on resolution made from field pattern observations.

However, with the theoretical limitation, Eq. (2), being of the same order, one may doubt whether these results reflect the limitations specific for the discrete structure. In order to make a reliable check, it is possible to study an artificial case of lower losses. Because of the logarithmic dependence in Eq. (2), we would need to assume the resistance a few orders of magnitude lower in order to improve dramatically the prediction of continuous model. In general, the overall characteristics of the lens, such as its impedance to the coil or transmission between the coil, remain qualitatively similar, however, the analysis is more complicated as all the resonant features are sharper and extra resonances emerge. We therefore restrict this consideration by assuming a ten times smaller dissipation. The frequency interval given for the imaging phenomena by the impedance criterion, is confined to
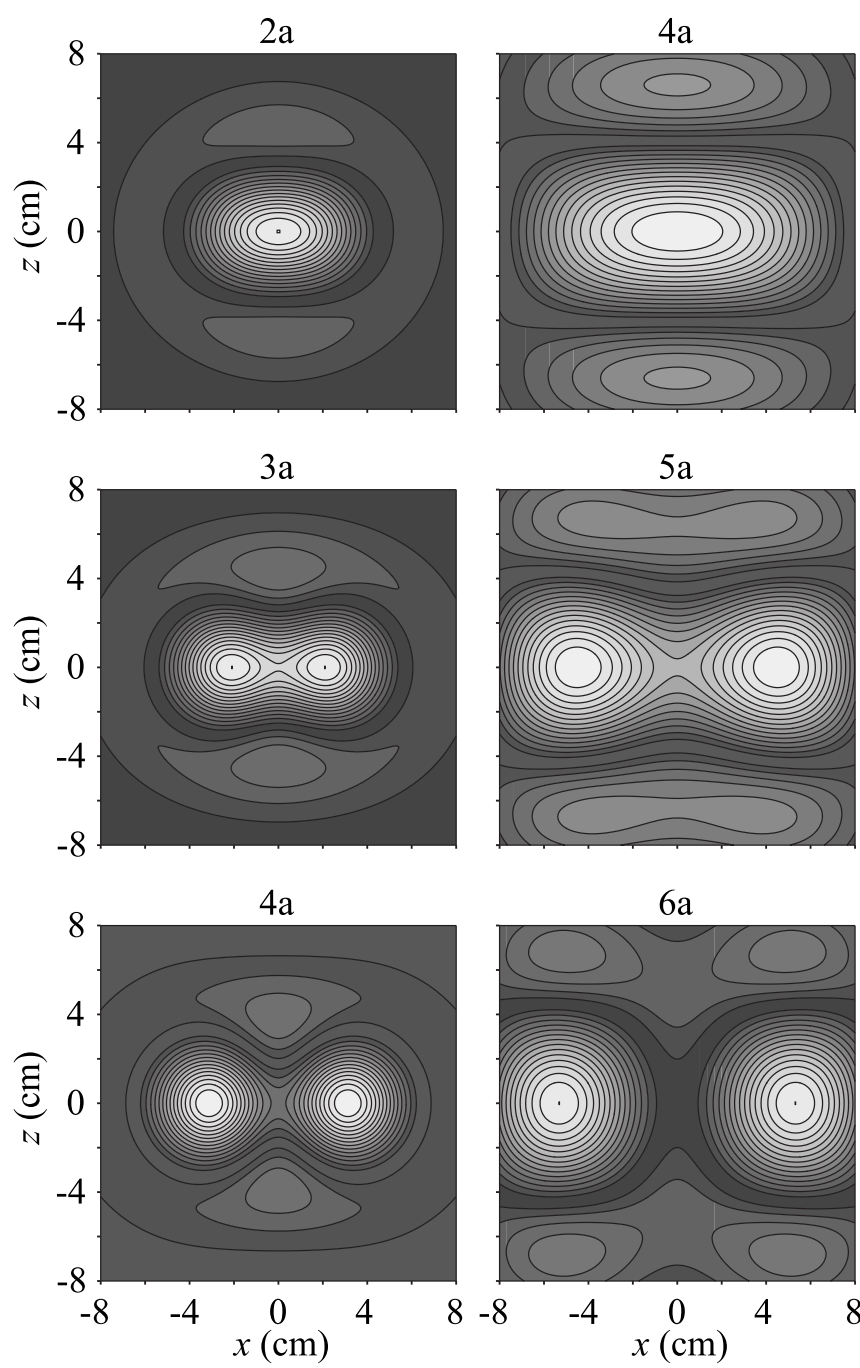

FIG. 9. Assessment of the resolution with a continuous slab model. Axial component $\left(H_{y}\right)$ of the total magnetic field observed in the image plane for the two point sources at various distances between each other (as shown above each image). Spatial harmonics are cut at either $300 \mathrm{~m}^{-1}$ (left column) or $65 \mathrm{~m}^{-1}$ (right column).

64.0-64.2 MHz while the transmission approach suggests 63.4-63.5 MHz. As to the field patterns in the image plane, further complication is brought by very rapid change in the patterns with frequency and numerous multiple additional maxima observed practically in the whole frequency range. By analyzing the patterns for $6 a$ separation between the two small sources, we were able to give preference to the frequency interval of 63.46 to $63.50 \mathrm{MHz}$, which is once again close to the transmission optimum. We should note, though, that such a favorable interval may appear different for different geometry, and is not very reliable. With this precaution, we performed a resolution check analogous to that of Fig. 7 at a frequency $63.48 \mathrm{MHz}$. It can be seen (Fig. 10) that although the patterns look much more "noisy" with extra excitations, the two sources can be reasonably distinguished at separations more than $5 a$ while at a smaller distance the central spot dominates. As for these losses resolution predicted by Eq. (2) is less than $3 a$, we feel confident that the 
$3 a$

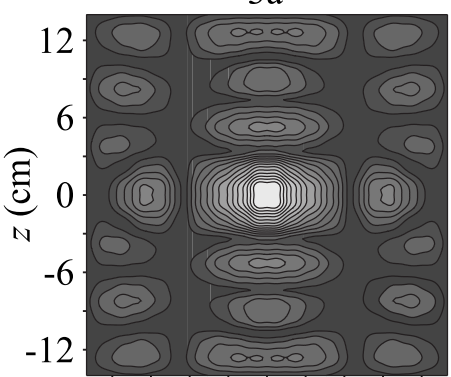

$4 \mathrm{a}$

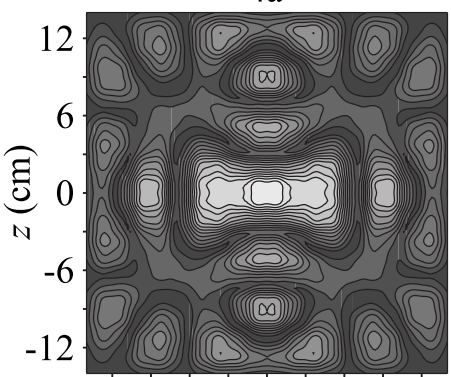

$5 \mathrm{a}$

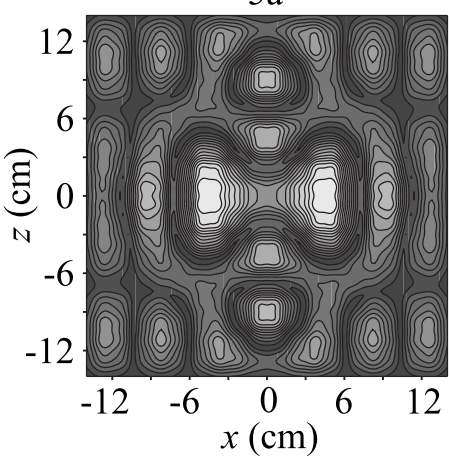

$6 a$

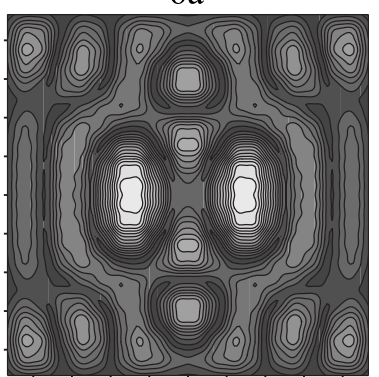

$8 \mathrm{a}$

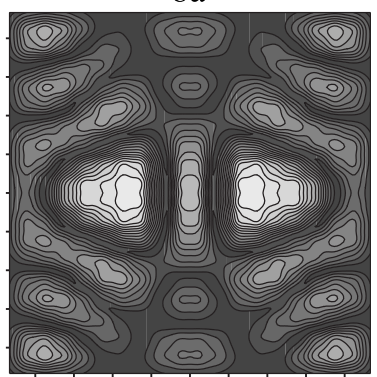

$12 \mathrm{a}$

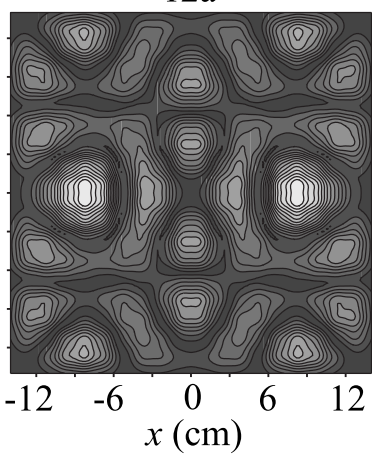

FIG. 10. Axial component $\left(H_{y}\right)$ of the total magnetic field observed (at 63.6 MHz) in the image plane for the two small sources (coils of $5 \mathrm{~mm}$ radius) placed in the source plane at various distances between each other. Dissipation in the lens is assumed to be ten times smaller as compared to Fig. 7

observed limitation should be attributed to the discreteness effect.

On the other hand, with low losses the above patterns of magnetic field are very sensitive to subtle variations in frequency and setup geometry. The additional peaks can be comparable or even exceed the image peaks in magnitude. This is especially remarkable when the coils are placed nonsymmetrically with respect to the lens center (Fig. 11): while with the realistic losses the shifted sources can be well resolved, the patterns at low loss are not comprehensive and could not be improved even by frequency variation. This is a consequence of numerous magnetoinductive excitations (spatial resonances) across the lens structure ${ }^{25,26}$ which are favored by low dissipation and relatively small size of the entire lens. This leads to various standing wave patterns which depend on frequency and coil parameters. In other words, a clear analogy to the phenomena, predicted for homogenized media, are not possible here because of the strong spatial dispersion. ${ }^{17,20,21}$ For these reasons, we feel that it is
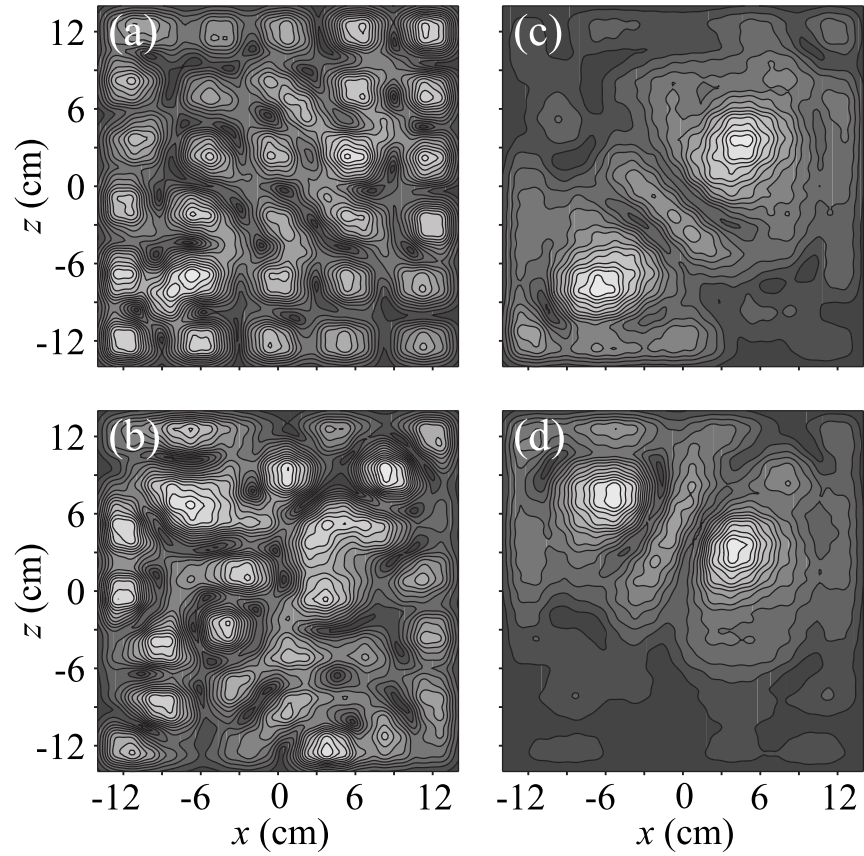

FIG. 11. Axial component $\left(H_{y}\right)$ of the total magnetic field observed (at $63.6 \mathrm{MHz}$ ) in the image plane for the two small sources (coils of $5 \mathrm{~mm}$ radius) placed nonsymmetrically in the source plane: one source is at $x_{1}=3 a$ and $z_{1}=2 a$ from the lens center, the other one is at either [(a) and (c)] $x_{1}=-4 a, z_{1}=-5 a$ (distance approximately $10 a$ ) or $\left[(\mathrm{b})\right.$ and (d) $x_{1}=-4 a, z_{1}=5 a$ (distance approximately 7.5a). Comparison between the low-loss case [(a) and (b)] and realistic dissipation $[(\mathrm{c})$ and (d)].

impossible to discuss consistently lens resolution at low losses, as the whole structure does not perform as intended with the original design. Note that the presence of such inhomogeneities is also noticeable for realistic losses (Fig. 7) but in that case they are largely suppressed and do not interfere so significantly with the image patterns.

Nevertheless we can conclude that the spatial resolution is not improved by decreased dissipation and that a reasonable distinction between the small sources is only possible for the same separations as with realistic losses. Therefore, resolution is indeed severely limited by discreteness of the lens and cannot be made better than five lattice constants.

Finally, we should comment that the result which we obtained with regards to resolution, is consistent with the effective medium theory. Indeed, it was shown explicitly for CLR metamaterials that the characteristic length of response formation in the bulk is of the order of few lattice constants. ${ }^{19}$ This is also generally expected in condensed matter theory. ${ }^{31,32}$ Consequently, it does not make sense to speak of any effective material properties on a smaller scale and any excitations delivered on the level of individual elements are spread out across the corresponding area encompassing numerous unit cells. This makes an important distinction to the channeling approach ${ }^{12}$ to imaging: resolution on the level of lattice constant is only possible with an array of weakly interacting elements. When, however, metamaterial is build up as an internally coupled effective medium, the limitations discussed in this paper are unavoidable. 


\section{CONCLUSIONS}

We have shown that the behavior of a realistic metamaterial lens (which can be practically implemented with a limited number of capacitively loaded resonators), differs significantly from the predictions based on a continuous medium approximation. It turns out that no direct coincidence to the imaging properties of a $\mu=-1$ slab can be obtained with such a practical lens, although analogous phenomena, suitable for imaging applications, are available in a certain frequency range.

We have also assessed the resolution, achievable with a discrete lens and conclude that it is generally limited by 5-7 lattice constants. It is important to emphasize that this limitation is entirely structural and cannot be improved by decreasing losses.
The discussed peculiarities are enforced by discrete structure and finite size of the lens, and are thus in agreement with the restrictions for the classical effective medium theory. At the same time, rich phenomena observed for a practical lens, leave much room for further exploration and can be useful for various applications.

\section{ACKNOWLEDGMENTS}

This work was supported by the Spanish Ministerio de Educación y Ciencia and European Union FEDER funds (Projects No. TEC2007-65376, No. TEC2007-68013-C0201, and No. CSD2008-00066), by Junta de Andalucía (Project No. TIC-253), and by Czech Grant Agency (Project No. 102/09/0314).
${ }^{1}$ L. Solymar and E. Shamonina, Waves in Metamaterials (Oxford University Press, New York, 2009).

${ }^{2}$ R. Marqués, F. Martín, and M. Sorolla, Metamaterials with Negative Parameters (Wiley, New York, 2008).

${ }^{3}$ M. Lapine and S. Tretyakov, IET Microwaves, Antennas Propag. 1, 3 (2007).

${ }^{4}$ A. Sihvola, Metamaterials 1, 2 (2007).

${ }^{5}$ J. B. Pendry, Phys. Rev. Lett. 85, 3966 (2000).

${ }^{6}$ E. Shamonina and L. Solymar, Metamaterials 1, 12 (2007).

${ }^{7}$ E. Shamonina, V. A. Kalinin, K. H. Ringhofer, and L. Solymar, Electron. Lett. 37, 1243 (2001).

${ }^{8}$ S. Maslovski, S. Tretyakov, and P. Alitalo, J. Appl. Phys. 96, 1293 (2004).

${ }^{9}$ F. Mesa, M. J. Freire, R. Marqués, and J. D. Baena, Phys. Rev. B 72, 235117 (2005).

${ }^{10}$ M. J. Freire and R. Marqués, Appl. Phys. Lett. 86, 182505 (2005).

${ }^{11}$ M. J. Freire, R. Marqués, and L. Jelinek, Appl. Phys. Lett. 93, 231108 (2008).

${ }^{12}$ P. Ikonen, P. A. Belov, C. R. Simovski, and S. I. Maslovski, Phys. Rev. B 73, 073102 (2006).

${ }^{13}$ M. C. K. Wiltshire, J. V. Hajnal, J. B. Pendry, D. J. Edwards, and C. J. Stevens, Opt. Express 11, 709 (2003).

${ }^{14}$ X. Radu, D. Garray, and C. Craeye, Metamaterials 3, 90 (2009).

${ }^{15}$ R. R. A. Syms, I. R. Young, and L. Solymar, Metamaterials 4, 1 (2010).

${ }^{16}$ R. R. A. Syms, L. Solymar, and I. R. Young, Metamaterials 2, 122 (2008).

${ }^{17}$ C. Simovski, Metamaterials 2, 169 (2008).

${ }^{18}$ M. G. Silveirinha, Phys. Rev. B 75, 115104 (2007).
${ }^{19}$ M. Gorkunov, M. Lapine, E. Shamonina, and K. H. Ringhofer, Eur. Phys. J. B 28, 263 (2002).

${ }^{20}$ J. D. Baena, L. Jelinek, R. Marqués, and M. Silveirinha, Phys. Rev. A 78, 013842 (2008).

${ }^{21}$ M. G. Silveirinha, J. D. Baena, L. Jelinek, and R. Marqués, Metamaterials 3, 115 (2009).

${ }^{22}$ L. Jelinek, R. Marqués, and M. J. Freire, J. Appl. Phys. 105, 024907 (2009).

${ }^{23}$ M. J. Freire, L. Jelinek, R. Marques, and M. Lapine, J. Magn. Reson. 203, 81 (2010).

${ }^{24}$ M. Lapine, L. Jelinek, R. Marqués, and M. J. Freire, IET Microwaves, Antennas Propag. 4, 1132 (2010).

${ }^{25}$ E. Shamonina, V. A. Kalinin, K. H. Ringhofer, and L. Solymar, J. Appl. Phys. 92, 6252 (2002).

${ }^{26}$ O. Zhuromskyy, E. Shamonina, and L. Solymar, Opt. Express 13, 9299 (2005).

${ }^{27}$ O. Sydoruk, M. Shamonin, A. Radkovskaya, O. Zhuromskyy, E. Shamonina, R. Trautner, C. J. Stevens, G. Faulkner, D. J. Edwards, and L. Solymar, J. Appl. Phys. 101, 073903 (2007).

${ }^{28}$ O. Zhuromskyy, O. Sydoruk, E. Shamonina, and L. Solymar, J. Appl. Phys. 106, 104908 (2009).

${ }^{29}$ V. M. Agranovich and Yu. N. Gartstein, Metamaterials 3, 1 (2009)

${ }^{30}$ See supplementary material at http://link.aps.org/supplemental/ 10.1103/PhysRevB.82.165124 for frequency variation in magnetic field pattern.

${ }^{31}$ M. I. Ryazanov, Condensed Matter Electrodynamics (Nauka, Moscow, 1984).

${ }^{32}$ M. V. Gorkunov and M. I. Ryazanov, Sov. Phys. JETP 85, 97 (1997). 